\title{
ACTITUDES DEL DOCENTE COMÚN DE LA CIUDAD DE POSADAS FRENTE A LA INTEGRACIÓN DE LOS NIÑOS CON NECESIDADES EDUCATIVAS ESPECIALES
}

\author{
Edith Moroni y Gladys Duarte \\ Universidad Nacional de Misiones, Argentina
}

\begin{abstract}
RESUMEN
El objetivo de este trabajo fue analizar las actitudes de docentes de la escuela común de la ciudad de Posadas, Misiones, Argentina, frente a la integración de niños con Necesidades Educativas Especiales (NEE). Hasta el momento de la realización del presente estudio no se habían ofrecido cursos de capacitación sobre el tema. El análisis actitudinal giró en torno de dos posturas fundamentales: tolerancia y aceptación. Estas posturas se analizaron, junto con otras variables, entre docentes que habían recibido niños con NEE en sus aulas y quienes nunca tuvieron esta experiencia.

La información fue obtenida mediante una escala actitudinal y un cuestionario adaptados, originariamente elaborados y aplicados en Gran Bretaña y España. Con fines metodológicos, el instrumento fue sometido a un proceso de validación estadística. La muestra definitiva estuvo compuesta por 250 sujetos que fueron seleccionados al azar del total de docentes de la ciudad de Posadas, población previamente relevada.

Los datos obtenidos revelan actitudes más favorables entre los docentes que no han tenido niños integrados en sus aulas en comparación con los que ya han participado en programas de integración.

Un análisis cualitativo permitió identificar uno de los datos más significativos: la percepción que tiene el docente de su capacidad para esta tarea. Se reconocen déficit en los aspectos organizacionales, vocacionales, de capacitación especial, de formación profesional, entre otros, como justificativos de dicha percepción. Los resultados obtenidos son coincidentes con estudios similares realizados en otros países.

Este estudio exploratorio permitió la verificación de los factores más sobresalientes, altamente determinantes en la formación de actitudes hacia la integración: apoyo profesional específico dado por la maestra integradora, capacitación especial, grupos reducidos y grado de severidad.
\end{abstract}

Las profundas transformaciones de la educación actual impulsadas desde la legislación en nuestro país ponen de manifiesto la necesidad de rever diversos aspectos que hacen a la realidad educativa moderna. Uno de esos aspectos está relacionado con la propuesta de integración de niños que hasta hace unos años eran considerados discapacitados y actualmente son vistos como personas con necesidades educativas especiales (NEE). La propuesta de integración, que trata de incorporar a estos niños a las escuelas comunes, en la medida en que 


\section{MORONI Y DUARTE}

las condiciones particulares de cada caso lo permitan, requiere, por su parte, una gran reestructuración en diferentes ámbitos de la esfera escolar.

La intención de incorporar a los niños con NEE a las aulas de educación común responde a una tendencia mundial hacia la integración física, laboral y social de tales personas. De hecho, la Declaración de Salamanca de 1994 ha proclamado la necesidad de que los sistemas educativos de la diferentes naciones adopten programas a fin de llevar en cuenta la amplia diversidad de características y necesidades de sus alumnos. La escuela inclusiva parece ser uno de los medios más eficaces para disminuir las actitudes discriminatorias para con los niños con NEE.

A pesar de que las actitudes de las personas han sido tema de numerosos enfoques conceptuales, aún hoy no existe una definición de ellas con la cual todos concuerden. De hecho, existen varias definiciones de lo que se entiende por actitud, lo que implica un elemento distorsionante, ya que la ambigüedad del término puede llevar a confusiones. Según Baeza Hernández (1991), varios autores admiten tres componentes básicos presentes en toda actitud: (a) el componente cognitivo, definido como el conjunto de creencias de una persona, que incluyen juicios de valor acerca de un objeto y elaboradas en base a lo que se conoce de dicho objeto; (b) el componente afectivo, que implica el gusto o una respuesta emocional hacia un objeto que es vivenciado como placentero o displacentero y (c ) el componente conductual, que se refiere a toda inclinación a actuar de una manera determinada ante un objeto.

En la formación de las actitudes y, obviamente en la modificación de las mismas, intervienen diversos factores que son los que condicionan enorme- mente la manera de actuar de una persona ante un determinado objeto de actitud. Según Fishbein y Ajzen (citados en Baeza Hernández, 1991) "la conducta social humana no está determinada por motivos inconscientes, deseos irresistibles ni creencias caprichosas, sino que, por el contrario, el individuo considera las implicaciones de su acción antes de desempeñar una determinada conducta. El sujeto controla su propia conducta sirviéndose de la información que es la base de su decisión” (p. 79). Esta información disponible por el sujeto (componente cognitivo) está poderosamente condicionada por su experiencia previa, además de los factores contextuales actuales que conforman la naturaleza de una actitud.

En esta línea de pensamiento, es obvio suponer que las actitudes de los docentes comunes para con el niño con NEE es la base que condicionaría el éxito o fracaso de todo plan de integración educativa de dichos niños.

Se han desarrollado diversas especulaciones para tratar de explicar las diferentes manifestaciones actitudinales que son subyacentes de las relaciones sociales entre personas "normales" y "diferentes”. Según Vitale (1994), “frente a un hombre concreto, entran a jugar como filtro de las percepciones, las valoraciones que a cada uno como hombre perteneciente a una determinada categoría social, la sociedad le ha asignado. Ello se da con anterioridad a la experiencia individual directa con las personas con las que se interactúa 'cara a cara' y signan la relación con ellas” (p. 39).

El abordaje conceptual del presente trabajo resalta dos tipos actitudinales cualitativamente diferentes, cuyas definiciones apuntan a una organización persistente de juicios acerca de un objeto o situación, condicionada por la historia personal y por las circunstancias presentes y que predisponen al sujeto en favor 


\section{ACTITUDES DEL DOCENTE COMÚN}

de respuestas preferidas: las actitudes de tolerancia y las de aceptación.

Las actitudes de tolerancia del sujeto evidencian el conocimiento de la presencia del niño con NEE en la sociedad pero no implican necesariamente una apertura para el acercamiento a él ni una modificación de la propia conducta que tienda a facilitar la comunicación con él. Este nivel actitudinal implica bajo grado de aceptación ya que no supone tendencia al establecimiento de relaciones sociales con sujetos en cuestión.

Las actitudes de aceptación implican un alto grado de tolerancia y acercamiento hacia la persona con NEE, por lo cual ésta es facilitadora del proceso de integración de esta persona a la sociedad. Las opiniones del sujeto revelan voluntad para modificar sus repertorios conductuales adaptándolos a las características del individuo especial.

No obstante, si bien la percepción social acerca de una problemática determinada influye en nuestras propias actitudes, está demostrado, mediante diversos estudios científicos en el área de la psicología, que las actitudes de las personas normales para con las personas “diferentes” están poderosamente condicionadas por el tipo y frecuencia de interacciones que cuentan en sus propias experiencias personales previas, además de los factores contextuales actuales que conforman una actitud determinada (Fishbein y Ajzen, citados en Baeza Hernández, 1991).

En la situación especial que los docentes viven en relación con la integración de niños con NEE, obviamente juegan un papel primordial los procesos actitudinales particulares hacia dichos niños, pero también, como ha sido demostrado por varios estudios, la percepción personal que tienen acerca de su propia preparación profesional para realizar una adecuada integración, lo cual constituye un factor extremamente condicionante de su actuación dentro del aula con dichos niños.

En ese sentido, Scruggs y Mastropieri (1996) han efectuado una amplia revisión de la literatura publicada entre 1958 y 1995. Esta revisión incluyó trabajos de investigación basados en escalas actitudinales y/o inventarios aplicados a 10.560 docentes de EE. UU., Australia y Canadá, un total de 28 informes publicados en diferentes revistas de divulgación científica. Dichos informes fueron correlacionados estadísticamente con el fin de establecer una correspondencia de los datos obtenidos en cada uno de ellos.

Los autores de este trabajo señalan que a pesar de la diversificación de escalas actitudinales, de inventarios y de las distintas áreas geográficas donde fueron aplicados, las respuestas de los docentes entrevistados fueron altamente equiparables. Según ellos, una de las conclusiones más relevantes extraídas del análisis de las diferentes investigaciones, es que la aceptación de niños con NEE para la integración dependería estrechamente de la severidad de la discapacidad de los alumnos en cuestión. Por otro lado, sólo cerca de un tercio de los docentes encuestados han admitido que poseen suficiente tiempo y suficiente preparación para atender a niños con NEE en sus aulas. Otro dato interesante que se deriva de este trabajo es la constatación de que la percepción de los docentes en cuanto a su capacitación para atender a estos niños no ha variado con el correr de las décadas que abarcó el relevamiento de la literatura. En relación a esto, los autores concluyen que la falta de mejoramiento de la percepción de los docentes de su propia preparación estaría indicando que los programas de formación docente son deficientes en cuanto a preparación para la integración.

En la misma línea de investigación, 


\section{MORONI Y DUARTE}

en un estudio sobre el efecto del entrenamiento de profesores sobre sus actitudes, Larrivee (citado en Baeza Hernández, 1991) comparó tres grupos de maestros sometidos a diversas condiciones de entrenamiento. Se constató que el grupo con mayor entrenamiento presentaba actitudes más positivas hacia la integración que los otros dos.

En nuestro medio, a pesar de que la integración se está desarrollando desde hace algunos años, todavía no se cuenta con evaluaciones globales y confiables de dichos proyectos. Cabe aclarar que estos proyectos no contaron con programas de capacitación a los docentes de las escuelas comunes interesados en el tema, antes de encomendarles los niños con NEE. Las escuelas especiales para integrar a los alumnos que están en condiciones de hacerlo, envían a una docente titulada en Educación Especial, cuyo rol es el acompañamiento tanto de niños con NEE como de docentes comunes que los aceptan en sus aulas; en esta provincia recibe el nombre de Maestra Integradora (MI, véase Anexo II). Se estima que tanto el conocimiento como las actitudes de los docentes comunes, junto con su percepción acerca de su preparación para atender a niños con NEE y de las condiciones generales para la implementación de dichos proyectos, son factores esenciales para una evaluación global de la eficacia de los programas de integración.

El presente trabajo, basado en las consideraciones anteriores, tiene por objetivo describir la situación del docente de la ciudad de Posadas ante la problemática de la integración escolar, comparándose un grupo de docentes que ya viene trabajando con niños con NEE con otro que nunca ha tenido contacto con ellos. Se supone que el contacto directo con esos niños puede ser un factor que podría determinar diferencias actitudina- les y evaluativas de la capacidad profesional de los docentes entre este grupo y el grupo conformado por los docentes que nunca han desarrollado actividades en estos proyectos.

\section{Sujetos \\ Método}

Los sujetos del presente trabajo fueron los docentes de escuelas comunes primarias del cono urbano de la ciudad de Posadas, Misiones, Argentina, en actividad en el año 1996.

Se utilizó un diseño probabilístico estratificado para la selección de la muestra a partir de un listado completo de establecimientos escolares ofrecido por el Consejo General de Educación de la Provincia de Misiones. Dicho listado permitió la realización de un relevamiento de todas las secciones de grado. En total, fueron relevadas 1551 secciones de grado que corresponden a los 84 establecimientos educativos que funcionaban en su momento en la ciudad de Posadas. Sin embargo, cabe aclarar que aproximadamente un tercio de la población docente trabajaba doble turno. Vale decir que el número total de secciones no se corresponde necesariamente con el número total de docentes. La selección de la muestra fue realizada en forma aleatoria a través de sorteo de todas las secciones de grado relevadas, obteniéndose una muestra final compuesta por 250 docentes, aproximadamente un 15\% del total de los docentes de la ciudad de Posadas. Una vez encuestados, los docentes fueron divididos en dos grupos: (a) grupo A, conformado por docentes que tuvieron experiencia con niños con NEE para su integración escolar, avalados o no por la Supervisión de Educación Especial, (b) grupo B: integrado por docentes de escuelas comunes que no tuvieron experiencia en el trabajo con niños con NEE. 


\section{ACTITUDES DEL DOCENTE COMÚN}

Las escuelas se clasificaron en tres categorías según las características de la población que atiende: (a) céntricas, (b) barriales y (c) periféricas.

\section{Instrumento}

El instrumento para la recolección de datos (ver Anexo I) constaba de dos secciones. La sección I indagó cuestiones referentes a algunos aspectos personales y profesionales de los docentes: (a) datos referidos a la función docente, (b) datos personales, (c) experiencias anteriores, (d) experiencia actual, (e) experiencias de colegas, (f) ítem de respuesta abierta. La sección II estuvo formada por una escala actitudinal de tipo Likert. Se ha creído conveniente la replicación y su consecuente validación de la escala actitudinal utilizada por Baeza Hernández (1991) en la ciudad de Murcia, España, la que, a su vez, es una replicación de la utilizada por Larrivee y Cook (1979 ), compuesta por 29 ítemes que contienen sentencias de opinión que abarcan ocho dimensiones relativas a las actitudes de los docentes hacia la integración (Baeza Hernández, 1991): (a) la educación en general, (b) la filosofía de la integración, (c) los efectos que produce la integración en los aspectos: social, emocional y cognitivo de los niños normales, (d) los efectos que produce la integración en los aspectos social, emocional y cognitivo de los niños con NEE, (e) la conducta en clase de los niños con NEE, (f) el funcionamiento cognitivo de los niños con NEE, (g) los padres de los niños con NEE, y (h) las habilidades con las que se perciben los docentes para desarrollar sus trabajos con niños con NEE.

Todos los ítemes fueron adaptados de su versión original, debido a las diferencias idiomáticas. Para la validación del instrumento, se eligieron 50 jueces (docentes en actividad) representativos de la muestra seleccionada, a los cuales se les administraron las dos secciones del instrumento de medición.

Se adjudicó una valoración positiva o negativa a cada ítem según éste reflejase una actitud favorable o desfavorable hacia la integración, dentro de las ocho dimensiones. Así, por ejemplo, dentro de la dimensión "conducta en clase del niño con NEE” un ítem relacionado sería el número 9, en el cual se enuncia que la “conducta de los niños con NEE sería un mal ejemplo para los otros niños”. Este ítem fue valorado como negativo ya que si el sujeto entrevistado se manifestase de acuerdo con la afirmación estaría, en realidad, expresando una opinión desfavorable hacia la integración de estos niños.

En el siguiente paso se procedió a la suma de los valores obtenidos para cada sujeto y a su ordenación de mayor a menor, hecho lo cual, se descartó el 50\% central de la distribución para trabajar únicamente con los cuartiles 1 y 4. Es decir, que se trabajó con 24 jueces, el $25 \%$ definitivamente favorable y el $25 \%$ claramente desfavorable; con estos jueces se realizó el control de cada uno de los ítemes para seleccionar los más discriminatorios, para cuyo fin se aplicó la $t$ de Student a los efectos de comprobar en cuáles de ellos las diferencias de medias entre ambos cuartiles resultaba estadísticamente significativa.

Se seleccionaron los 17 ítemes que arrojaron las diferencias de medias más significativas $(p<.10)$, los cuales constituyeron el instrumento de medición definitivo. Ellos son los siguientes: 1, 2, 4, 6, 7, 8, 9, 11, 12, 14, 16, 18, 21, 23, 24, 26 y 28 (ver Anexo I).

La escala, cuyo rango varía entre 17 puntos (actitud más desfavorable) y 85 puntos (actitud más favorable), permitió determinar las siguientes tres categorías: (a) favorable: 65 a 85 puntos, (b) neutra: 51 a 64 puntos, y (c) desfavorable: 17 a 


\section{MORONI Y DUARTE}

50 puntos.

\section{Procedimiento}

A partir de la conformación del diseño muestral, se procedió a la distribución de los protocolos a los sujetos seleccionados. En la mayoría de los casos fue entregado personalmente a los docentes, a excepción de algunas escuelas cuyas exigencias internas no permitían el acercamiento a los maestros en horario de clase. En dichos casos el material fue entregado a los directivos del establecimiento quienes se comprometían a distribuir a los docentes seleccionados.

En el momento de la entrega del material, se ofrecían algunas explicaciones muy sucintas acerca del objetivo del trabajo y se formulaba la consigna de trabajo, consistente de una somera descripción de la forma de completamiento de los cuestionarios. Cabe aclarar que los docentes tenían conocimiento del estudio que sería efectuado, gracias al relevamiento realizado con antelación, que permitió un contacto previo con ellos.

El material fue recogido en los mismos establecimientos educativos después de un lapso que varió entre 10 y 20 días, debido al gran número de escuelas participantes y al difícil acceso a las mismas en días de lluvia.

Del total de 250 protocolos distribuidos, 195 fueron considerados válidos. Los restantes fueron invalidados por distintas razones, entre ellas, por incompletos o porque los docentes no los devolvieron por haberse acogido a licencias reglamentarias.

\section{Resultados}

La sección I del instrumento permitió reunir la información que permite ofrecer una descripción de la muestra encuestada, de acuerdo con sus características relevantes.
Un 23\% de los docentes participantes se encontraba trabajando en escuelas céntricas, un $40 \%$ en escuelas barriales y un $37 \%$ en escuelas periféricas.

Según la dependencia de las escuelas, encontramos que el $75 \%$ de la muestra se desempeña en establecimientos de gestión pública y el 35\% restante en escuelas privadas, confesionales y no confesionales. Las secciones de grado más numerosas corresponden a algunas escuelas privadas.

La titulación de los docentes, su edad y antigüedad en el cargo también se tuvieron en cuenta. Hay un alto porcentaje (66\%) de Profesores para la Enseñanza Primaria, título que se otorgó en los últimos 25 años. En cuanto a la antigüedad en la docencia, un 29\% tenía menos de 5 años en el ejercicio de la docencia, un $61 \%$ entre 5 y 20 años y un 10\% más de 20 años. Esto nos muestra que hay un importante número de docentes con experiencia y fuerza para la tarea, con mayor porcentaje de jóvenes sobre los que piensan en su jubilación.

En cuanto al sexo, es realmente abrumadora (97\%) la cantidad de docentes de sexo femenino.

Referente a los docentes que tienen familiares con deficiencias, encontramos solamente el $15 \%$, generalmente sobrinos o hermanos.

Se clasificó la población de acuerdo a la posesión o no de experiencias con niños con NEE, comprobándose que el $56 \%$ de los encuestados no había recibido niños con NEE en sus secciones de grado.

Se solicitó a los docentes que efectuaran una evaluación acerca del éxito alcanzado en el proceso de integración de cada uno de los alumnos con NEE recibidos en sus clases. Se observó que un 30\% de los casos señaló haber obtenido un bajo rendimiento, un 42\% calificó al mismo como mediano y solo en el 


\section{ACTITUDES DEL DOCENTE COMÚN}

28\% de los casos consideraron haber alcanzado un alto éxito.

Relacionando el éxito alcanzado por alumno integrado de acuerdo a la recepción o no de apoyo, y en caso de haber contado con el mismo si este estuvo apoyado por una maestra integradora (MI), se observa que el contar con apoyo de profesionales implicó un mayor éxito en la tarea, que se acentúa notablemente con la asistencia de una MI (ver Tabla 1). La asociación entre ambas variables es muy significativa $\left(\mathrm{V}_{\mathrm{C}}=.108 ; \chi^{2}=\right.$ 22.805; $\mathrm{p}=.001$ ).

Consideramos que, si bien existía la posibilidad de una fuerte incidencia del apoyo recibido sobre el éxito alcanzado en las experiencias con niños con NEE,

Tabla 1

Éxito alcanzado por alumno según tipo de apoyo recibido

\begin{tabular}{lcccc}
\hline & \multicolumn{4}{c}{ Tipo de apoyo } \\
\cline { 2 - 5 } \multicolumn{1}{c}{$\begin{array}{c}\text { Éxito } \\
\text { alcanzado }\end{array}$} & $\begin{array}{c}\text { Sin } \\
\text { apoyo }\end{array}$ & $\begin{array}{c}\text { Apoyo } \\
\text { sin MI }\end{array}$ & $\begin{array}{c}\text { Apoyo } \\
\text { con MI }\end{array}$ & Total \\
n=24 & $\mathrm{n}=21$ & $\mathrm{n}=106$ \\
\hline Bajo & $42 \%$ & $21 \%$ & $5 \%$ & $30 \%$ \\
Medio & $38 \%$ & $63 \%$ & $33 \%$ & $42 \%$ \\
Alto & $20 \%$ & $16 \%$ & $62 \%$ & $28 \%$ \\
\hline
\end{tabular}

también era importante contrastarlo con las diferentes discapacidades. La Tabla 2 muestra los porcentajes de éxito obtenido en las discapacidades más frecuentes. Puede observarse que la discapacidad visual es la que obtiene el mayor éxito.

Con la información recogida en la sección II se procedió a la clasificación de los docentes encuestados de acuerdo con sus actitudes hacia la integración. Los docentes se distribuyeron de la siguiente manera: el 21\% con una actitud favorable, el 58\% en una situación inter- media que denominamos neutra, y por último el 21\% con una actitud desfavorable hacia la integración.

Tabla 2

Éxito alcanzado por alumno según tipo de discapacidad

\begin{tabular}{lccc}
\hline \multirow{2}{*}{ Discapacidad } & \multicolumn{3}{c}{ Éxito alcanzado } \\
\cline { 2 - 4 } & Alto & Medio & Bajo \\
\hline Auditiva & $15 \%$ & $40 \%$ & $45 \%$ \\
Visual & $33 \%$ & $11 \%$ & $56 \%$ \\
Motora & $10 \%$ & $52 \%$ & $38 \%$ \\
R.M. leve & $32 \%$ & $52 \%$ & $16 \%$ \\
Conducta & $32 \%$ & $63 \%$ & $5 \%$ \\
Total & $23 \%$ & $48 \%$ & $29 \%$ \\
\hline
\end{tabular}

Según nuestro marco conceptual, existen dos tipos claros de actitud frente a la integración: (a) una de tolerancia y (b) otra de aceptación. Relacionando estos tipos con la estratificación de las actitudes en la escala, se asumió que entre los agrupados en las categorías neutra y desfavorable se encontrarían los que tendrían una tendencia a una actitud de tolerancia, en tanto que agrupados bajo la categoría favorable estarían los docentes con una tendencia a la actitud de aceptación.

La actitud docente hacia la integración de niños con NEE fue relevada independientemente de las experiencias del docente, pero retomando la posesión o no de la misma se observa que en aquellos sin experiencia hay una leve inclinación favorable, es decir una mayor aceptación que entre aquellos que han tenido experiencia (ver Tabla 3), sin que la asociación haya llegado a ser estadísticamente significativa $\left(\mathrm{V}_{\mathrm{C}}=.056 ; \chi^{2}\right.$ $=.604 ; \mathrm{p}=.7393)$.

Entre los docentes encuestados con experiencia con niños con NEE, el 39\% 


\section{MORONI Y DUARTE}

Tabla 3

Actitud hacia la integración de acuerdo con la experiencia con niños con NEE

\begin{tabular}{lccc}
\hline & \multicolumn{3}{c}{ Experiencia con niños con NEE } \\
\cline { 2 - 4 } & $\begin{array}{c}\text { Con expe- } \\
\text { riencia } \\
(\mathrm{n}=85)\end{array}$ & $\begin{array}{c}\text { Sin expe- } \\
\text { riencia } \\
\text { Actitud }\end{array}$ & $\begin{array}{c}\text { Total } \\
(\mathrm{n}=110)\end{array}$ \\
\hline Favorable & $18 \%$ & $23 \%$ & $21 \%$ \\
Neutra & $60 \%$ & $57 \%$ & $58 \%$ \\
Desfavorable & $22 \%$ & $20 \%$ & $21 \%$ \\
\hline
\end{tabular}

declaró no haber contado con apoyo en la tarea, en tanto el $29 \%$ tuvo el apoyo de profesionales relacionados a las discapacidades en cuestión y el 32\% recibió apoyo con MI independientemente de otros. Si bien esto se registró por alumno, hasta tres alumnos como máximo por docente, para trabajar el tipo de apoyo se consideró: (a) apoyo con MI, cuando al menos en algunos de los alumnos contó con ello; (b) sin apoyo, cuando en ninguna de las experiencias recibió apoyo especializado; y (c) apoyo sin MI, son aquellos casos que tuvieron apoyo profesional sin MI (ver Tabla 4).

Un análisis de la actitud en relación al tipo de apoyo recibido o no apoyo muestra que existe una leve asociación entre ambas variables. El coeficiente de asociación empleado fue la V de Cramer, que arrojó un valor de 0.281. Se observa que la actitud de los docentes ante la integración tiende a ser más favorable entre los que contaron con apoyo en la tarea a la vez que se nota una mayor diferencia cuando interviene la MI, no apareciendo entre éstos ningún caso como desfavorable. La asociación encontrada entre las variables en cuestión es estadísticamente significativa $\left(\mathrm{V}_{\mathrm{C}}=\right.$ $\left..552 ; \chi^{2}=14.943 ; \mathrm{p}=.0006\right)$.
Tabla 4

Actitud de integración según tipo de apoyo

\begin{tabular}{lcccc}
\hline & \multicolumn{4}{c}{ Tipo de apoyo } \\
\cline { 2 - 5 } & $\begin{array}{c}\text { Sin } \\
\text { apoyo } \\
\text { Actitud }\end{array}$ & $\begin{array}{c}\text { Apoyo } \\
\text { sin MI } \\
(\mathrm{n}=33)\end{array}$ & $\begin{array}{c}\text { Apoyo } \\
\text { con MI } \\
(\mathrm{n}=24)\end{array}$ & $\begin{array}{c}\text { Total } \\
(\mathrm{n}=126)\end{array}$ \\
\hline $\mathrm{n}=193)$
\end{tabular}

No obstante la valoración de los apoyos recibidos en la integración, como adecuados, es de destacar que al relacionarlos con la actitud, vemos que sólo un 21\% poseen una actitud favorable (ver Tabla 5).

La Tabla 6 muestra la disposición por parte de los docentes con experiencia de integración a repetir la misma, ya sea con modificaciones o no. Si bien existe una débil asociación entre ambas variables $\left(\mathrm{V}_{\mathrm{C}}=0.337\right)$, se observa que entre los que lo harían de la misma forma, hay una tendencia a tener una actitud menos desfavorable que entre aquellos que proponen modificaciones. Entre los docentes que declararon que no repetirían la experiencia no existe ningún docente con actitud favorable $(p=.04)$.

Tabla 5

Actitud hacia la integración de acuerdo con la valoración de los apoyos recibidos

\begin{tabular}{lccc}
\hline & \multicolumn{3}{c}{ Valoración del apoyo } \\
\cline { 2 - 4 } Actitud & $\begin{array}{c}\text { Adecuado } \\
(\mathrm{n}=47)\end{array}$ & $\begin{array}{c}\text { Inadecuado } \\
(\mathrm{n}=2)\end{array}$ & $\begin{array}{c}\text { Total } \\
(\mathrm{n}=49)\end{array}$ \\
\hline Favorable & $21 \%$ & -- & $21 \%$ \\
Neutra & $70 \%$ & -- & $67 \%$ \\
$\begin{array}{l}\text { Desfavo- } \\
\text { rable }\end{array}$ & $9 \%$ & $100 \%$ & $12 \%$ \\
\hline
\end{tabular}




\section{ACTITUDES DEL DOCENTE COMÚN}

Tabla 6

Actitud hacia la integración según repetición de experiencia

\begin{tabular}{|c|c|c|c|c|}
\hline \multirow[b]{2}{*}{ Actitud } & \multicolumn{4}{|c|}{ Repetición de experiencia } \\
\hline & $\begin{array}{c}\text { De la } \\
\text { misma } \\
\text { forma } \\
(n=15)\end{array}$ & $\begin{array}{c}\text { Con } \\
\text { modifi- } \\
\text { cacio- } \\
\text { nes } \\
(\mathrm{n}=19)\end{array}$ & $\begin{array}{l}\text { No re- } \\
\text { petiría } \\
(n=10)\end{array}$ & $\begin{array}{c}\text { Total } \\
(\mathrm{n}=44)\end{array}$ \\
\hline Favorable & $20 \%$ & $21 \%$ & -- & $16 \%$ \\
\hline Neutra & $73 \%$ & $58 \%$ & $40 \%$ & $59 \%$ \\
\hline $\begin{array}{l}\text { Desfavo- } \\
\text { rable }\end{array}$ & $7 \%$ & $21 \%$ & $60 \%$ & $25 \%$ \\
\hline
\end{tabular}

entre los que lo harían de la misma forma, hay una tendencia a tener una actitud menos desfavorable que entre los que proponen modificaciones. Entre los docentes que declararon que no repetirían la experiencia no existe ningún docente con actitud favorable $(\mathrm{p}=.04)$.

Algunos docentes formularon comentarios de colegas, de cómo les había resultado la experiencia obtenida. Se relacionaron las respuestas con las actitudes de los docentes encuestados y se obtuvieron los siguientes resultados (ver Tabla 7).

Tabla 7

Relación de la actitud con el relato de experiencias de colegas

\begin{tabular}{|c|c|c|c|}
\hline \multirow[b]{2}{*}{ Actitud } & \multicolumn{3}{|c|}{$\begin{array}{c}\text { Valoración de experiencias de } \\
\text { colegas }\end{array}$} \\
\hline & $\begin{array}{c}\text { Positiva } \\
(n=74)\end{array}$ & $\begin{array}{c}\text { negativa } \\
(n=17)\end{array}$ & $\begin{array}{c}\text { Total } \\
(n=91)\end{array}$ \\
\hline Favorable & $26 \%$ & $6 \%$ & $22 \%$ \\
\hline Neutra & $66 \%$ & $35 \%$ & $60 \%$ \\
\hline Desfavorable & $8 \%$ & $59 \%$ & $59 \%$ \\
\hline
\end{tabular}

Se observa un mayor porcentaje de docentes con actitud favorable el grupo que recibió comentarios positivos. La asociación entre ambas variables es moderada $\left(\mathrm{V}_{\mathrm{C}}=0,404 ; \mathrm{p}<.001\right)$.

\section{Discusión}

Entre las características de los docentes relevados y las respuestas obtenidas a los ítemes planteados para medir la actitud de los mismos frente a la integración, resulta llamativo el porcentaje de respuestas en la categoría neutra, lo que podría deberse a diferentes razones, algunas de ellas ligadas, por ejemplo, a un vacío de conocimiento sobre el tema, a una falta de vocación para esta tarea; a una falta de compromiso con niños que presentan características diferentes para aprender, al hecho de considerar que son patrimonio de las escuelas especiales y al temor a situaciones desconocidas, entre otras.

Estas interpretaciones se sustentan en diversas concepciones teóricas acerca de las “actitudes”. Según Allport (citado en Hollander, 1982), las actitudes se adquieren por aprendizaje y actúan como energías motivacionales impulsando al sujeto hacia determinadas conductas, que pueden ser aversivas o propelentes hacia un objeto, evento y/o situación. Muchos relatos de los docentes son consistentes con esta concepción de la actitud. A modo de ejemplo, un elevado número de ellos ha manifestado no sentirse capacitado y/o suficientemente informado como para trabajar con niños con NEE. Esto revela un desconocimiento del tema, que a su vez deviene de la pobre formación académica de los docentes, no siendo responsabilidad absoluta de ellos.

La educación especial se ha manejado en forma autónoma de la educación común durante décadas y en nuestro medio el tema de la integración es reciente, 


\section{MORONI Y DUARTE}

apareciendo la figura legal en la nueva Ley de Educación 24.125 vigente a partir de 1993. A pesar de ello, hay que reconocer que siempre existió "integración” de manera informal.

El análisis de las actitudes de docentes que manifestaron no haber tenido experiencia con alumnos con NEE y de los que señalaron que sí la habían tenido revela una tendencia hacia una actitud más favorable por parte de quienes no habían tenido contacto con estos niños. Estadísticamente no se ha observado una asociación entre estas variables.

Siguiendo esta línea de análisis, la variable apoyo general fue reconstruida a partir de los ítemes 19 y 24, en los que se indagaba por los tipos de apoyo recibidos. Es posible observar que, dentro del grupo de docentes que había tenido experiencia de integración, el grupo que manifestó falta de apoyo reveló actitudes más desfavorables; sin embargo el grupo de docentes que señaló haber recibido apoyo por parte de una MI, dentro del apoyo oficial, presentó actitudes más favorables y ninguna desfavorable, inclusive comparativamente con el grupo que recibió apoyo profesional de psicólogos, psicopedagogos o fonoaudiólogos, pero no de una MI. Aparentemente la figura de la MI es crucial para la integración de los niños con NEE, especialmente si se tiene en cuenta que cuando se realizó esta investigación los docentes comunes no habían recibido asesoramiento alguno. En este sentido esta figura actuaría como respaldo para los docentes y estaría disponible para cubrir las demandas de los mismos, lo cual le garantizaría una cierta seguridad en su accionar frente al alumno. Pero aún habría que realizar algunos ajustes, pues un tercio de estos docentes informaron que repetirían la experiencia con modificaciones (véase en el Anexo II el rol de la MI).

Entre los docentes que reciben apoyo profesional, no oficial, se observan actitudes más desfavorables.

En el ítem "Repetiría la experiencia" casi la cuarta parte respondió negativamente, lo que podría interpretarse como que sus experiencias en este sentido fueron negativas, lo que tiene mayor sustento en el hecho de que un $60 \%$ tiene actitudes desfavorables, el $40 \%$ "neutro" y ningún docente de este grupo presenta actitudes favorables. Dentro del grupo que repetiría la experiencia, una mayor proporción lo "repetiría con modificaciones". La frecuencia de las actitudes favorables de ese grupo es la misma que la de las desfavorables. Para los que repetirían la experiencia de la "misma forma" un mayor porcentaje presenta actitudes favorables.

Estos datos reforzarían la afirmación de que las experiencias anteriores positivas provocan actitudes favorables, mientras que por otro lado las experiencias negativas inducen al sujeto hacia actitudes desfavorables, lo cual tiene relación con el concepto de actitud cuyo contenido se considera que resume los conocimientos más relevantes que se hayan adquirido en contactos pasados con el objeto.

Un apoyo profesional adecuado y el éxito alcanzado por el alumno integrado constituyen, también, factores de influencia en la formación de actitudes positivas hacia la integración. Estos factores son comparativamente más determinantes en la aceptación de la integración que la escasa preparación que los docentes comunes reconocen poseer.

Se ha pensado que los relatos de colegas podrían influir en la actitud del docente. De hecho los datos demuestran que cuando el relato de la experiencia de colegas es positivo hay una tendencia actitudinal más favorable. Por otro lado cuando el relato de la experiencia es negativo, la actitud es más desfavorable. Estos datos parecen indicar que el relato 


\section{ACTITUDES DEL DOCENTE COMÚN}

de los colegas influye en las actitudes de los docentes en forma significativa.

En el relevamiento de secciones realizado, fue posible la constatación de manera informal, en conversaciones directas con los docentes, de la fuerte preocupación que tienen por dos grandes cuestiones que deben enfrentar: (a) por un lado, el cada vez más generalizado problema de indisciplina, que llega a agobiar al personal de las instituciones escolares, y (b) por otro lado, la imposibilidad de revertir las dificultades de aprendizaje, causa de la principal deserción del alumnado del primer ciclo, focalizados principalmente en las escuelas de la periferia.

A partir de todas las informaciones recogidas en este estudio, se puede percibir que el docente siente que las actuales condiciones del sistema educativo, incluyendo la infraestructura edilicia, los recursos materiales y humanos, además del aspecto remunerativo, no son favorables para la implementación de un proyecto de integración.

Algunos resultados de la presente investigación concuerdan en gran medida con aquellos obtenidos por diferentes estudios realizados en otros países (Scruggs y Mastropieri, 1996).

\section{Conclusiones}

Este estudio exploratorio permitió la verificación de algunos factores más sobresalientes que son altamente determinantes en la formación de actitudes hacia la integración. Ellos pueden ser agrupados de la siguiente manera:

1. Apoyo profesional específico, brindado por la MI. Los docentes necesitan contar con el asesoramiento y supervisión constante de algún profesional que actúe en el ámbito de la educación especial.

2. Capacitación especial. Los docentes comunes necesitan contar en su formación de grado, con un abordaje más profundo y específico de los tópicos fundamentales de la educación especial.

3. Grupos reducidos. Los docentes necesitan que los grupos de alumnos sean reducidos cuando se incluyen niños con NEE en los mismos, según las respuestas abiertas (ítem 29).

Para finalizar, es importante resaltar que el Ministerio de Educación de la Provincia de Misiones tuvo en cuenta esta investigación para reorganizar proyectos de integración que incluyeron algunos cursos de perfeccionamiento docente y la modificación del rol de la MI.

\section{Referencias}

Baeza Hernández, C. (1991). La actitud del profesor hacia la integración escolar; un estudio de la región de Murcia. Murcia: Caja de Ahorro.

Consejo General de Educación. (1995). Proyecto de integración de personas con alguna discapacidad al sistema educativo común en sus diferentes modalidades y niveles en la Provincia de Misiones. Posadas: Supervisión de Educación Especial.

Hollander, E. (1982). Principios y métodos de psicología social. Buenos Aires. Amorrortu.

Scruggs, T. y Mastropieri, M. (1996). Teacher perceptions of mainstreming/inclusion, 19581995: A research synthesis. Exceptional Children, 63(1), 59-74.

Vitali, María. (1994). Identidad social del discapacitado: tendencias básicas. Posadas: Facultad de Humanidades y Ciencias Sociales, UNAM. 


\section{MORONI Y DUARTE}

\section{A N E X O I \\ INSTRUMENTO \\ UN ESTUDIO DE LA OPINIÓN DE LOS MAESTROS EN RELACIÓN CON LA INTEGRACIÓN DE LOS NIÑOS CON NECESIDADES EDUCATIVAS ESPECIALES}

La reciente legislación requiere que los niños con necesidades especiales, sean integrados en las clases regulares con la extensión que cada integración sea posible.

El propósito de este cuestionario es obtener la información de lo que usted piensa sobre la problemática de la integración escolar. El cuestionario es anónimo; se asegura el carácter confidencial y un serio tratamiento de cuantos datos y respuestas se obtengan.

Por favor, atienda a las normas de contestación antes de responder a las preguntas.

AGRADECEMOS DE ANTEMANO SU ATENCIÓN

\section{SECCIÓN I}

Por favor, responda marcando una $X$ en los paréntesis correspondientes a la respuesta deseada. Si se equivoca, marque con un círculo la respuesta incorrecta y marque de nuevo con una $X$ la respuesta correcta.

I) Escuela donde trabaja y datos referidos a su función docente (Si trabaja doble turno o más, responda sobre la situación en la escuela donde se le entregó esta encuesta.)

1. Trabaja:
( ) un turno
( ) doble turno ( ) triple turno

2.Ubicación de su escuela:
( ) centro de la ciudad
( ) barrio urbano
( ) barrio periférico

3. Categoría de escuela donde trabaja:
( ) pública
( ) privada confesional
( ) privada no confesional
( ) cooperativa

4. Titulación académica que posee:
( ) Maestro Normal
( ) Profesor para Enseñanza Primaria
( ) Maestra Jardinera
( ) Profesor en Psicopedagogía
( ) Profesor en Psicología
( ) Otra:

5. Grado o nivel en el que imparte clase:
( ) Nivel Inicial
( ) Primer Ciclo EGB
( ) Segundo Ciclo EGB
( ) Tercer Ciclo EGB

6. Número de alumnos de su clase:
( ) de 16 a 20 alumnos
( ) de 21 a 25 alumnos
( ) de 26 a 30 alumnos
( ) de 31 a 35 alumnos
( ) de 36 a 40 alumnos
( ) de más de 40 alumnos 


\section{ACTITUDES DEL DOCENTE COMÚN}

7. Señale la antigüedad docente:
( ) menos de 5 años
( ) de 5 a 10
() de 10 a 15
( ) de 15 a 20

( ) más de 20

\section{II) Datos personales}

8. Sexo:
( ) varón
( ) mujer

9. Edad:
( ) menos de 25
( ) de 25 a 35 años
( ) de 36 a 45 años
( ) más de 45 años

10. Estado civil:
() soltero/a
( ) casado/a
( ) viudo/a
( ) divorciado/a
( ) otra situación:

11. Número de hijos:
( ) de hasta 6 años
( ) de 7 a 14 años
( ) de 15 a 20 años
( ) de más de 20 años
( ) no tiene
Total:

12. Tiene algún hijo o familiar cercano que posea algún tipo de deficiencia: ( ) sí $\leadsto$ pasar a preguntas 13 y 14 . ( ) no $\leadsto$ pasar a pregunta 15.

13. Qué grado de parentesco:
( ) padres
( ) hermanos
( ) sobrinos
( ) tíos
( ) otros:

14. La deficiencia es de tipo:
( ) físico-motora
( ) psíquico-mental
( ) sensorial: sordo
( ) otras deficiencias:

III) Experiencias anteriores

15. ¿'Tuvo alumnos discapacitados integrados en años anteriores?

( ) no tuvo $\rightarrow$ (si marca ésta, pasar a pregunta 21)

( ) tuvo

16. En todas estas experiencias anteriores ¿cuál de estas discapacidades fue la más frecuente?

auditiva

Alumno 1

( )
Alumno 2

( )
Alumno 3 


\section{MORONI Y DUARTE}

$\begin{array}{llll}\text { visual } & (\text { ) } & (\text { ) } & (\text { ) } \\ \text { motor } & (\text { ) } & (\text { ) } & (\text { ) } \\ \text { retardo mental leve } & (\text { ) } & (\text { ) } & (\text { ) } \\ \text { conducta } & (\text { ) } & (\text { ) } & (\text { ) } \\ \text { otras: } & (\text { ) } & (\text { ) } & (\text { ) }\end{array}$

17. Califique su grado de éxito en experiencias anteriores con niños con NEE

$\begin{array}{llll}\text { muy bajo } & (\text { ) } & (\text { ) } & (\text { ) } \\ \text { bajo } & (\text { ) } & (\text { ) } & (\text { ) } \\ \text { mediano } & (\text { ) } & (\text { ) } & (\text { ) } \\ \text { alto } & (\text { ) } & (\text { ) } & (\text { ) } \\ \text { muy alto } & (\text { ) } & (\text { ) } & (\text { ) }\end{array}$

18. ¿Dispuso de apoyos específicos?

\begin{tabular}{lccc}
\multicolumn{1}{c}{ Alumno 1} & Alumno 2 & Alumno 3 \\
no dispuso de ningún apoyo ( ) & ( ) & ( ) \\
dispuso de apoyo privado & $($ ) & $($ ) & $($ ) \\
dispuso de apoyo oficia & $($ ) & $($ ) & $($ ) \\
dispuso de ambos & $($ ) & ( ) & ( )
\end{tabular}

19. ¿Qué tipo de apoyos?

$\begin{array}{lccc} & \text { Alumno } 1 & \text { Alumno } 2 & \text { Alumno } 3 \\ \text { servicio psicopedagógico } & (\text { ) } & (\text { ) } & (\text { ) } \\ \text { fonoaudiológico } & (\text { ) } & (\text { ) } & (\text { ) } \\ \text { psicomotricidad } & (\text { ) } & (\text { ) } & (\text { ) } \\ \text { psicológico } & (\text { ) } & (\text { ) } & (\text { ) } \\ \text { fisioterapéutico } & (\text { ) } & (\text { ) } & (\text { ) } \\ \text { maestra ntegradora } & (\text { ) } & (\text { ) } & (\text { ) }\end{array}$

20. En caso de haber contado con apoyos específicos ¿cómo los valoraría?
( ) adecuados
( ) inadecuados

\section{IV) Experiencia actual con niños con necesidades educativas especiales}

21. ¿Actualmente tiene alumnos con alguna discapacidad integrados?

( ) sí $\rightarrow$ (responder preguntas 22 a 26)

( ) no $\leftrightarrow$ (pasar a pregunta $\left.n^{\circ} 27\right)$

22. ¿Quién hizo la derivación del alumno?

Alumno 1

supervisión especial

( )

dirección de la escuela

()

familia del alumno

()

otros

( )

23. ¿Dispone de apoyos específicos?

Alumno 1

$\begin{array}{cc}\text { Alumno } 2 & \text { Alumno } 3 \\ \text { ( ) } & (\text { ) } \\ \text { ( ) } & (\text { ) } \\ \text { ( ) } & (\text { ) } \\ \text { ( ) } & (\text { ) }\end{array}$

dispone de ningún apoyo

( )

Alumno 2

Alumno 3

( )

( ) 


\section{ACTITUDES DEL DOCENTE COMÚN}

dispone de apoyo privado

( )

()

dispone de apoyo oficial

( )

dispone de ambos

( )

( )

( )

()

( )

24. ¿Qué tipo de apoyos?

Alumno 1 Alumno $2 \quad$ Alumno 3

servicio psicopedagógico

( )

fonoaudiológico

( )

psicomotricidad

psicológico

fisioterapéutico

( )

maestra ntegradora

( )

( )

()

( )

( )

( ) ( )

( ) ( )

( ) ( )

( ) ( )

25. En caso de haber contado con apoyos específicos ¿cómo los valoraría?
( ) adecuados
( ) inadecuados

26. ¿Repetiría la experiencia con niños con necesidades educativas especiales
( ) de la misma forma
( ) con modificaciones

( ) no la repetiría

\section{V) Experiencias de colegas}

27. ¿Conoce colegas que hayan vivido la experiencia de integración?
( ) sí
( ) no

28. En caso afirmativo, la experiencia relatada fue:
( ) positiva
( ) negativa

29. ¿Tiene algo más que expresar sobre este tema? Por favor, hágalo a continuación.

\section{SECCIÓN II}

Por favor, rodee el número que esté de acuerdo con su opinión, siguiendo la escala expuesta a continuación. La mejor respuesta es la que refleja honestamente su opinión.
Escala: $\quad$ 1. Muy en desacuerdo
2. En desacuerdo
3. Indeciso.
4. De acuerdo.
5. Muy de acuerdo.

\begin{tabular}{|l|l|c|c|c|c|c|}
\hline & & MD & D & I & A & MA \\
\hline 1 & $\begin{array}{l}\text { Muchas de las cosas que los maestros hacen con los estudiantes } \\
\text { normales en una clase son apropiadas para niños con necesidades } \\
\text { especiales. }\end{array}$ & 1 & 2 & 3 & 4 & 5 \\
\hline 2 & $\begin{array}{l}\text { Las necesidades de los alumnos discapacitados pueden satisfacerse } \\
\text { mejor de forma especial en clases separadas. }\end{array}$ & 1 & 2 & 3 & 4 & 5 \\
\hline
\end{tabular}




\section{MORONI Y DUARTE}

\begin{tabular}{|c|c|c|c|c|c|c|}
\hline 3 & $\begin{array}{l}\text { La conducta de un niño con necesidades especiales, en una clase, } \\
\text { requiere más paciencia por parte del profesor que la que demanda un } \\
\text { niño normal. }\end{array}$ & 1 & 2 & 3 & 4 & 5 \\
\hline 4 & $\begin{array}{l}\text { El desafío de integración en una clase común fomentaría el desarrollo } \\
\text { académico de los niños con necesidades especiales. }\end{array}$ & 1 & 2 & 3 & 4 & 5 \\
\hline 5 & $\begin{array}{l}\text { La atención extra requerida por los niños con necesidades especiales } \\
\text { iría en perjuicio de los otros alumnos. }\end{array}$ & 1 & 2 & 3 & 4 & 5 \\
\hline 6 & $\begin{array}{l}\text { La integración ofrece interacción entre grupos mixtos, los cuales } \\
\text { favorecen el entendimiento y la aceptación de las diferencias. }\end{array}$ & 1 & 2 & 3 & 4 & 5 \\
\hline 7 & $\begin{array}{l}\text { Es difícil mantener el orden en una clase en la que se hallan niños con } \\
\text { necesidades especiales. }\end{array}$ & 1 & 2 & 3 & 4 & 5 \\
\hline 8 & $\begin{array}{l}\text { Los maestros de clases comunes poseen el alto grado de experiencia } \\
\text { necesaria para trabajar con alumnos con necesidades especiales. }\end{array}$ & 1 & 2 & 3 & 4 & 5 \\
\hline 9 & $\begin{array}{l}\text { La conducta de los niños con necesidades especiales sería un mal } \\
\text { ejemplo para los otros niños. }\end{array}$ & 1 & 2 & 3 & 4 & 5 \\
\hline 10 & $\begin{array}{l}\text { El aislamiento en una clase específica tiene un efecto negativo en el } \\
\text { desarrollo social y emocional de un alumno con necesidades especiales. }\end{array}$ & 1 & 2 & 3 & 4 & 5 \\
\hline 11 & $\begin{array}{l}\text { El niño con necesidades especiales probablemente desarrollaría } \\
\text { habilidades académicas más rápidamente en una clase especial que en } \\
\text { una clase normal. }\end{array}$ & 1 & 2 & 3 & 4 & 5 \\
\hline 12 & $\begin{array}{l}\text { La mayoría de los niños con necesidades especiales no hacen un } \\
\text { esfuerzo adecuado para completar sus tareas. }\end{array}$ & 1 & 2 & 3 & 4 & 5 \\
\hline 13 & $\begin{array}{l}\text { Laint } \\
\text { signif }\end{array}$ & 1 & 2 & 3 & 4 & 5 \\
\hline 14 & mporta bien & 1 & 2 & 3 & 4 & 5 \\
\hline 15 & $\begin{array}{l}\text { Los } m \\
\text { enseñ }\end{array}$ & 1 & 2 & 3 & 4 & 5 \\
\hline 16 & $\begin{array}{l}\text { El con } \\
\text { perjud }\end{array}$ & 1 & 2 & 3 & 4 & 5 \\
\hline 17 & $\begin{array}{l}\text { Los alumnos con necesidades especiales absorben el tiempo del } \\
\text { maestro. }\end{array}$ & 1 & 2 & 3 & 4 & 5 \\
\hline 18 & $\begin{array}{l}\text { ación escolar con niños con necesidades especiales, promove- } \\
\text { egración social. }\end{array}$ & 1 & 2 & 3 & 4 & 5 \\
\hline 19 & iales exhiba una & 1 & 2 & 3 & 4 & 5 \\
\hline 20 & $\begin{array}{l}\text { sea dado por los } \\
\text { lase normal. }\end{array}$ & 1 & 2 & 3 & 4 & 5 \\
\hline 21 & $\begin{array}{l}\text { La integración de al } \\
\text { puede ser beneficios }\end{array}$ & 1 & 2 & 3 & 4 & 5 \\
\hline 22 & $\begin{array}{l}\text { Los niños con } \\
\text { mente de "que }\end{array}$ & 1 & 2 & 3 & 4 & 5 \\
\hline 23 & $\begin{array}{l}\text { La integración tiene posibilidad de } \\
\text { desarrollo emocional del niño con ne }\end{array}$ & 1 & 2 & 3 & 4 & 5 \\
\hline 24 & $\begin{array}{l}\text { El niño con necesidades especiales estaría socialmente aislado de los } \\
\text { demás niños normales de la clase. }\end{array}$ & 1 & 2 & 3 & 4 & 5 \\
\hline 25 & $\begin{array}{l}\text { Los padres de un niño con necesidades especiales, no presentan } \\
\text { mayores problemas para el maestro de clase común que los padres de } \\
\text { un niño normal. }\end{array}$ & 1 & 2 & 3 & 4 & 5 \\
\hline 26 & $\begin{array}{l}\text { La integración de niños con dificultades especiales necesitaría mayor } \\
\text { perfeccionamiento de los profesores de clases comunes. }\end{array}$ & 1 & 2 & 3 & 4 & 5 \\
\hline 27 & $\begin{array}{l}\text { A los niños con necesidades especiales se le darían todas las oportuni- } \\
\text { dades de funcionamiento en la clase común, si fuera posible. }\end{array}$ & 1 & 2 & 3 & 4 & 5 \\
\hline 28 & $\begin{array}{l}\text { Es posible que los niños con necesidades especiales creen confusión } \\
\text { en una clase común. }\end{array}$ & 1 & 2 & 3 & 4 & 5 \\
\hline 29 & $\begin{array}{l}\text { La presencia de niños con necesidades especiales promovería acepta- } \\
\text { ción de las diferencias por parte de los alumnos normales. }\end{array}$ & 1 & 2 & 3 & 4 & 5 \\
\hline
\end{tabular}




\section{ACTITUDES DEL DOCENTE COMÚN}

\section{ANEXO II \\ Documento elaborado por la Supervisión de Educación Especial de la Provincia de Misiones en "Apoyo y asesoramiento a docentes de escuelas especiales”}

\section{ROL DE LA MAESTRA INTEGRADORA}

Para la Maestra Integradora, integrar implica:

- Acompañar y sostener al niño en su integración a la Escuela Común que ha sido preparado adecuadamente en la Escuela Especial.

Acompañar y sostener significa:

1. Establecer vínculos con la familia del niño.

2. Establecer vínculos con la Escuela que lo recibe, directivos, docente que lo tiene a cargo y resto del plantel docente.

- Con el docente se trabaja semanalmente a través de entrevistas durante las cuales se intercambian estrategias en relación a las distintas áreas pedagógicas, como así también las áreas sociales, afectivas y cognitivas.

- Estas entrevistas pueden ir variando en frecuencia, según la necesidad de cada caso.

- La metodología varía para cada alumno teniendo en cuenta áreas específicas afectadas por su discapacidad particular, de ahí que se hable de las necesidades educativas especiales.

3. Algunas estrategias empleadas:

- Trabajar los objetivos desarrollados por la Maestra del grado en apoyos pedagógicos en turno contrario dentro de la misma Escuela Común.

- Planificar objetivos específicos pertinentes a cada especialidad (discapacidad neurológica-ciegos o amblíopes-sordos o hipoacúsicos-discapacidad mental) y trabajarlos en entrevistas con el alumno en contraturno en Escuela Especial o dentro de la Escuela Común o Centro especializado.

- La frecuencia de los encuentros con los alumnos será acordado desde el Equipo de Integración, la familia y/o niño o joven.

- Asistencia del Maestro Integrador a Actos escolares de la Escuela Común que considere necesarios para así observar la participación del niño, su conducta social, respeto a las normas y otros aspectos difíciles de percibir en situación áulica cotidiana.

- Asistencia del Maestro Integrador y/o Equipo de Integración a algunas reuniones de personal en la Escuela Común para informar, orientar, concientizar e intercambiar ideas acerca del proceso de Integración.

4. La duración del proceso de Integración depende de cada caso (1 año o varios). El corte se va dando en forma gradual, disminuyendo la frecuencia del apoyo, las entrevistas, manteniendo continuos diálogos con la familia, el alumno y el docente.

5. El trabajo es una tarea en equipo, conjuntamente con los integrantes del equipo psicotécnico o equipo escolar encargado de la Integración desde la Escuela Especial, los que se reúnen semanalmente para la resolución de las diferentes situaciones planteadas.

6. El Maestro Integrador elabora su horario de trabajo según el horario de las Escuelas Comunes (diurnas o nocturnas), según el horario de las Maestras Comunes (horas especiales, recreos, etc.), según el horario libre de las familias y las disponibilidades del niño en el tiempo en que no va a la escuela. Por lo tanto no siempre coincide con el mismo horario y/o turno de la Escuela Especial.

7. SER UN AGENTE DE TRANSFORMACIÓN EDUCATIVA. 